\title{
Contrast-enhanced ultrasonography in the initial evaluation of the kidney graft function: a pilot study.
}

\author{
Florin Ioan Elec ${ }^{1,2}$, Alina Daciana Elec1, Sorana D. Bolboacă ${ }^{3}$, Mihai Adrian Socaciu ${ }^{4,5}$, \\ Adriana Milena Muntean', Gheorghiță Iacob', Tudor Moisoiu ${ }^{2,4}$, Razvan Zaro4, Alexandra- \\ Ioana Andrieș ${ }^{4}$, Maria Ramona Bădulescu², Radu Mihai Ignat ${ }^{4,6}$, Radu Ion Badea,5
}

${ }^{1}$ Clinical Institute of Urology and Renal Transplantation, ${ }^{2}$ Department of Urology, "Iuliu Haţieganu” University of Medicine and Pharmacy, ${ }^{3}$ Department of Medical Informatics and Biostatistics, "Iuliu Hațieganu" University of Medicine and Pharmacy, ${ }^{4}$ Department of Medical Imaging, "Iuliu Haţieganu" University of Medicine and Pharmacy, ${ }^{5}$ Department of Medical Imaging, „Prof. Dr. Octavian Fodor ”Regional Institute of Gastroenterology ${ }^{6}$ Department of Anatomy, "Iuliu Hațieganu” University of Medicine and Pharmacy, Cluj-Napoca, Romania

\begin{abstract}
Aim: Our study aimed to assess the usefulness of contrast-enhanced ultrasound (CEUS) in the initial evaluation of the graft function. Materials and method: A cross-sectional study was conducted in the early postoperative period on patients with kidney transplantation, between September 2017 to November 2018. Two groups of patients were investigated; delayed graft function (DGF) and early graft function (EGF). All patients were examined by grey scale, Doppler ultrasound and CEUS. Results: Nineteen patients, aged from 23 to 64 years (mean age 50 years), 7 in the DGF group and 12 in the EGF group were evaluated. The resistive index (RI) show significantly higher values in the DGF group at the level of upper interlobar artery $(p=0.025)$ and medium interlobar artery $(p=0.02)$. The CEUS investigation shows a greater region of interest (ROI) area $(p=0.02)$ and lower values for wash-out area under the curve (WoAUC) $(p=0.047)$ and respectively wash-in and wash-out area under the curve (WiWoAUC) $(\mathrm{p}=0.031)$ for the DGF group. The quality of fit (QoF) proved lower in the DGF group either for evaluation of global graft $(\mathrm{p}=0.012)$, cortex $(\mathrm{p}=0.025)$, or medulla $(\mathrm{p}=0.009)$. A significant relationship among all patients was found between the glomerular filtration rate (GFR) $[\mathrm{ml} / \mathrm{min}]$ and the renal artery fall time (FT) $[\mathrm{s}](\mathrm{p}=0.012)$, WoAUC [a.u.] ( $\mathrm{p}=0.03$ ), and WiWoAUC [a.u.] ( $\mathrm{p}=0.024)$. The arterial QoF [\%] was associated with the arterial ROI area $(\mathrm{p}=0.048)$. Conclusions: Intensity CEUS parameters WoAUC and WiWoAUC may be useful to diagnose and follow-up grafts with delayed function. Additional studies on larger cohorts are required for the recommendation of CEUS as a routine evaluation of the transplanted kidney.
\end{abstract}

Keywords: kidney transplantation; graft function; ultrasonography; Doppler ultrasound; Contrast-Enhanced Ultrasonography (CEUS)

\section{Introduction}

Due to the ability to evaluate microvascular perfusion of the graft, contrast enhanced ultrasound (CEUS)

Received 15.12.2019 Accepted 25.03.2020

Med Ultrason

2020, Vol. 22, No 3, 272-278

Corresponding author: Gheorghiță Iacob

11/20 Arinilor street, 400568 Cluj-Napoca, Romania

E-mail: iacob.gheorghita@gmail.com

Phone: 0722984742 is credited with higher sensitivity and specificity. CEUS changes are able to precede the changing of the resistivity (RI) and pulsatility index measured by Doppler ultrasound [1]. Currently, the early post-transplant renal graft function is defined by serum creatinine on the $5^{\text {th }}$ postoperative day (POD5), the dialysis requirement and the presence of diuresis [2-4]. Ischemia-reperfusion generates a series of pathologic events, leading to acute kidney injury through ischemic injury and altered repair mechanisms $[5,6]$. Early graft function (EGF) is defined as serum creatinine $(\mathrm{sCr})$ lower than $3.0 \mathrm{mg} / \mathrm{dL}$ by POD5 and 
delayed graft function (DGF) is defined as the need for dialysis within the first week after transplantation [2-4]. The diagnosis of acute renal graft dysfunction is based on increased serum creatinine, decreased diuresis or proteinuria $[2,7]$. The Doppler ultrasound is commonly used to evaluate the renal graft. Although it is an accessible and non-invasive imaging method, it appears to have a limited utility in diagnosing the graft dysfunction due to the lack of specificity and a late appearance of Doppler ultrasound pathologic findings [8]. CEUS offers a multitude of quantitative parameters correlated directly to microvascular perfusion of the graft [9]. Kidney allograft assessment by CEUS mainly focuses on graft rejection, with a small number of studies on the evaluation of delayed (DGF) or early function (EGF) [10].

Our study aimed to assess the usefulness of CEUS in the early evaluation of the graft function with a special focus on a possible predictor for the indication of a biopsy protocol of the graft. Subsequently we were interested in the assessment of the relation between resistive index (RI) and CEUS parameters for patients with DGF or EGF.

\section{Material and methods}

A cross-sectional study was conducted in the postoperative period on a cohort of patients with kidney transplants at the Clinical Institute of Urology and Renal Transplantation Cluj-Napoca from October 2017 to November 2018. All transplanted recipients in the specified period who agreed to participate in the study, were included in the study. The patients were divided into two groups according to the allograft function: EGF and DGF. EGF was defined as serum creatinine $(\mathrm{sCr})<3.0$ $\mathrm{mg} / \mathrm{dL}$ by POD5. DGF was defined as the need for dialysis within the first week after transplantation.

The patients were assessed in terms of age, sex, body mass index (BMI), cause of the chronic kidney disease, atheromatous grade, $\mathrm{sCr}$, glomerular filtration rate (GFR) based on Modification of Diet in Renal Disease equation, protein/creatinine ratio, kidney dimensions and volume, RI and time-intensity curve (TIC) derived parameters on CEUS.

Evaluation of the graft by ultrasound was done with a General Electric Logiq E9 machine with a broad spectrum (1.5-6 MHz) convex probe (GE C1-6-D). The ultrasonography protocol included: a) setting for abdominal mode of the machine; b) identification of the graft; c) selection of most representative pictures in long and short axis, measurement of parenchymal thickness, cortical thickness, long axis, short axis and volume; d) evaluation of presence of blood flow by color and power Doppler and B flow mode; e) evaluation of RI in the interlobar ar- teries at the upper, middle and inferior levels; $f$ ) setting of the contrast mode (with simultaneous representation of grey scale and harmonic information on the dual display; mechanical index - 0.09-0.11; one focus point located posteriorly to the kidney); g) injection of contrast medium (SonoVue ${ }^{\circledR}$ - $1.6 \mathrm{ml}$ i.v. in bolus), followed by 10 $\mathrm{ml}$ saline solution flush into a peripheral vein at the arm; h) continuous evaluation in real time of the CEUS aspects for 2 minutes, with fixed long axis section through the kidney, assessing main arterial branches, cortex enhancement and medullar enhancement [11]; i) off-line quantification of the data using VueBox6.1 (Bracco), a dedicated software for tracing and evaluation of time intensity curves (TIC). To obtain TICs, we used cine loops with a length of 120 seconds from the moment of contrast injection, saved as RAW DICOM files. For each case we selected 4 regions of interest (ROI) occupying the entire renal parenchyma, inside a segmental artery, in the cortex and in the medulla. After plotting the TIC, different parameters were measured: peak enhancement (PE), wash in area under curve (WiAUC), rise time (RT), mean transit time local (mTT-TI), Time To Peak (TTP), Wash-in Rate (WiR), Wash-in Perfusion Index (WiPI)(WiAUC/ RT), wash-out AUC [AUC (TTP:TO)], wash-in and wash-out AUC (WiAUC+WoAUC), fall time (FT)(TOTTP) and quality of fit of the model to measured values.

The study was approved by the local Ethics Committee, according to the World Medical Association Declaration of Helsinki regarding Ethical Principles for Medical Research Involving Human Subjects. All patients gave informed consent for enrolment in the study.

\section{Statistical analysis}

Statistical analysis was made under the assumption that data do not follow the normal distribution since the sample sizes of the groups were less than 30 patients. Median and interquartile range $[(\mathrm{Q} 1$ to $\mathrm{Q} 3)$, where $\mathrm{Q}$ is the value of the quartile] were used to summarize quantitative data and the groups were compared with MannWhitney test. Number and percentages were reported for qualitative data and the frequencies were compared with Fisher exact test since values less than five were in the expected tables. The association analysis was conducted with Spearman's rank correlation method. Statistical analysis was done at a significance level of 5\% and all p-values less than 0.05 were considered statistically significant. Statistical analytics software (v.8, StatSoft, OK, USA) was used for statistical analysis.

\section{Results}

The main characteristics of the 19 patients included in study are detailed in Table I. The age of donor varied 
Table I. Main characteristics of the patients

\begin{tabular}{|c|c|c|c|c|}
\hline Characteristic & All, $n=19$ & DGF, $n=7$ & EGF, $n=12$ & p-values \\
\hline Age (years) & $50(39$ to 55$)$ & $53(49.5$ to 55.5$)$ & $43(37.5$ to 52.8$)$ & 0.236 \\
\hline Men & $11(57.9)$ & $4(57.1)$ & $7(58.3)$ & 0.366 \\
\hline Body Mass Index (BMI, kg/m²) & $29(25.5$ to 30.5$)$ & $31(29.5$ to 32$)$ & $26(24.5$ to 29.3$)$ & 0.007 \\
\hline $\begin{array}{l}\text { BMI class } \\
\text { Normal weight } \\
\text { Overweight } \\
\text { Obesity }\end{array}$ & $\begin{array}{l}3(15.8) \\
8(42.1) \\
8(42.1)\end{array}$ & $\begin{array}{l}0(0) \\
5(71.4) \\
2(28.6)\end{array}$ & $\begin{array}{l}3(25) \\
3(25) \\
6(50)\end{array}$ & 0.031 \\
\hline $\begin{array}{l}\text { Disease } \\
\text { Chronic glomerulonephrites } \\
\text { Nephropathy } \\
\text { Polycystic disease } \\
\text { Chronic kidney disease }\end{array}$ & $\begin{array}{l}6(31.6) \\
3(15.8) \\
8(42.1) \\
2(10.5)\end{array}$ & $\begin{array}{l}2(28.6) \\
1(14.3) \\
3(42.8) \\
1(14.3)\end{array}$ & $\begin{array}{l}4(33.3) \\
2(16.7) \\
5(41.7) \\
1(8.3)\end{array}$ & 0.977 \\
\hline $\begin{array}{l}\text { Atheromatous grade } \\
\text { Absent/minimum } \\
\text { Mild }\end{array}$ & $\begin{array}{l}11(57.9) \\
8(42.1)\end{array}$ & $\begin{array}{l}3(42.9) \\
4(57.1)\end{array}$ & $\begin{array}{l}8(66.7) \\
4(33.3)\end{array}$ & 0.229 \\
\hline Creatinine, $\mathrm{mg} / \mathrm{dL}$ & $1.75(1.29$ to 3.87$)$ & $6.7(3.9$ to 8.9$)$ & $1.3(1.2$ to 1.5$)$ & 0.0005 \\
\hline Glomerular Filtration Rate, $\mathrm{ml} / \mathrm{min}$ & $42(16.5$ to 59.3$)$ & $9(5$ to 16.5$)$ & $58.3(42.8$ to 64.7$)$ & 0.0005 \\
\hline Proteinuria/Creatininuria & $0.379(0.192$ to 0.637$)$ & $0.5(0.3$ to 0.725$)$ & $0.350(0.163$ to 0.483$)$ & 0.472 \\
\hline
\end{tabular}

The results are expressed by number (\%) or median (interquartile range, Q1 to Q3). DGF: delayed graft function; EGF: early graft function; BMI: body mass index

from 27 to 72 years, median $=51$ years ( 37.5 to 63.5$), 12$ men and 7 women, 4 cases of suicide, 2 of craniocerebral traumatism and 8 cases of stroke. For five donors, the cause of death was not recorded. Seven patients were with DGF and 12 with EGF. No significant difference was observed regarding the age of donor among groups $(p=0.446)$. All donors of the patients in the DGF group were men $(\mathrm{p}=0.015)$.

The CEUS evaluation was done in the timeframe from 5 to 90 days, without significant difference regarding the time from kidney transplantation to CEUS examination between EGF and DGF group ( $p=0.108)$. Ultrasound appearance of a kidney graft with EGF versus DGF are summarized below.

In the case of grafts with EGF (fig 1), we found in all cases normal echogenicity and good cortico-medullar differentiation in grey-scale mode and normal vessels distribution towards the cortex periphery on Doppler mode. On CEUS, the cortico-medullary phase shows a quick and homogeneous enhancement in the cortex while time-intensity curves (green=global kidney, yellow $=$ cortex, magenta=medulla) reveal a quick and steady enhancement in both cortex and medulla, with the peak in the medulla appearing later, with less intensity. On the CEUS color map, the distribution of the parameter WiWoAUC appears with higher values (red) in the cortex.

In all kidneys with DGF (fig 2) a slight increase in the size of the kidney and a hypoechoic, less corticomedullar differentiation was found. Color Doppler mode showed less vessels in the cortical periphery, with irregular trajectories. Higher RI was found at the level of the upper and medium interlobar artery compared to the EGF group, On CEUS, the cortico-medullary phase appeared late and with less intense cortical enhancement, especially in the periphery. TIC (green=global kidney, yellow $=$ cortex, magenta $=$ medulla) showed quick cortical enhancement, less intense than the mean in the whole kidney with a quick washout, followed a few seconds later by a double peak in the medulla, with less intensity. A second medullar peak was interpreted as the contrast recirculation through vascular shunts. A color map showed the distribution of the parameter WiWoAUC, with lower values (green) in the peripheral cortex, suggesting a decreased vascular volume. ROI area for the global kidney was higher $(\mathrm{p}=0.02)$ with significantly lower values for WoAUC $(\mathrm{p}=0.047)$ and respectively WiWoAUC $(p=0.031)$ compared with the EGF group. The only CEUS parameter with significant smaller values in the DGF group compared to the EGF group was the quality of fit QoF (Table II).

A significant association between RI at the upper interlobar artery and cortical TTP was identified for the DGF group $(\rho=-0.76, p=0.049$ ). Several significant associations between the US, Doppler US and CEUS were identified for EGF group (Table III).

Significant relationships were found between the renal artery FT, WoAUC and the WiWoAUC and GFR. The arterial QoF proved to be associated with the arterial ROI area used (Table IV). 


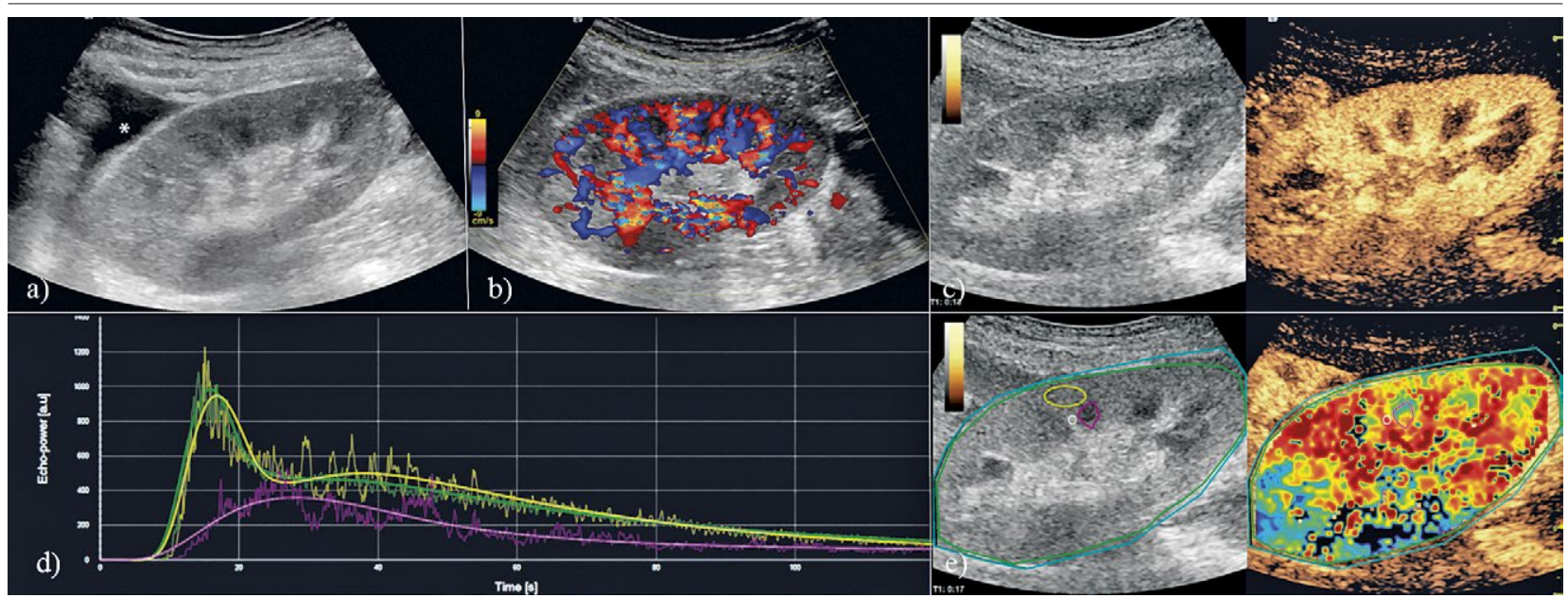

Fig 1. Ultrasound appearance of a kidney with early graft function: a) Grey-scale mode showing normal echogenicity and good cortico-medullar differentiation; note a small fluid collection near the upper pole $(*)$ b) Color Doppler mode showing a good vessel distribution towards the cortex periphery; c) CEUS image in the cortico-medullary phase showing a quick and homogeneous enhancement in the cortex; d) Time-intensity curves (green= global kidney, yellow=cortex, magenta=medulla) show a quick and steady enhancement in both cortex and medulla, with the peak in the medulla appearing later, with less intensity; e) color map showing the distribution of the parameter wash-in and wash-out AUC, with higher values (red) in the cortex.

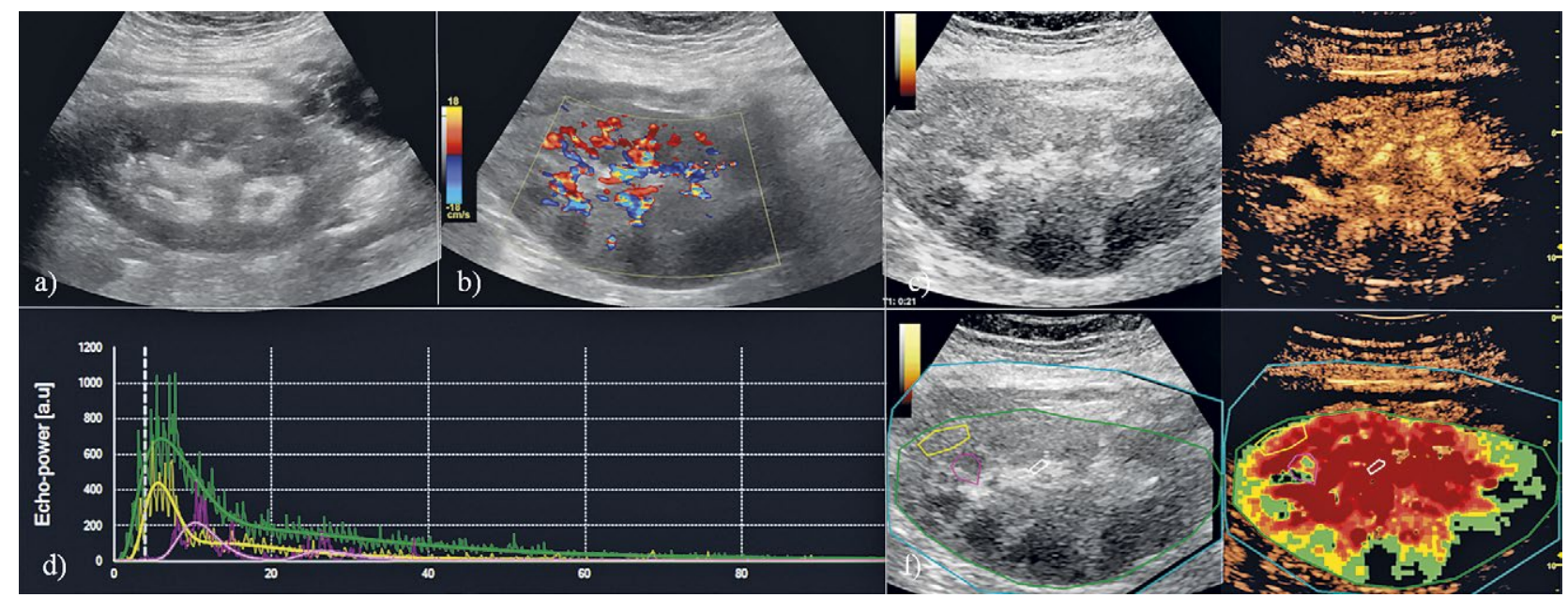

Fig 2. Ultrasound appearance of a kidney with a delayed graft function. a) Grey-scale mode showing a kidney slightly increased in size and hypoechoic, with less cortico-medullar differentiation; b) Color Doppler mode showing less vessels in the cortical periphery, with irregular trajectories; c) CEUS image in the cortico-medullary phase showing a late and less intense cortical enhancement, especially in the periphery; d) Time-intensity curves (green=global kidney, yellow $=$ cortex, magenta=medulla) show a quick cortical enhancement, less intense than the mean in the whole kidney with a quick washout, followed a few seconds later by a double peak in the medulla, with less intensity; the second medullar peak may be due to contrast recirculation through vascular shunts e) color map showing the distribution of the parameter wash-in and wash-out AUC, with lower values (green) in the peripheral cortex, suggesting a decreased vascular volume.

\section{Discussion}

CEUS has proven its value over the gray scale and Doppler US in the early postoperative evaluation of microcirculation of the renal grafts. The procedure is noninvasive, nontoxic and cheaper than computed tomography [12]. CEUS delivers functional data in real time with a very high accuracy in imaging superficial structures with a high level of vascularization. [13]. Even though it requires special software and expensive contrast agent, this technique can assess the microvascular tissue perfusion in patients with impaired renal function, without the need of an experienced investigator [14].

Assessment of renal graft microperfusion using CEUS might be better correlated with the underlying processes which occur during rejection and acute tubular 
Table II. Main grey scale, Doppler and contrast enhanced ultrasound characteristics of the investigated sample

\begin{tabular}{|c|c|c|c|c|}
\hline Characteristic & All $n=19$ & DGF $n=7$ & EGF $n=12$ & p-values \\
\hline Volume, $\mathrm{cm}^{3}$ & $199(156.5$ to 235.5$)$ & 243 (204 to 293.5$)$ & $179.5(137.0$ to 210.8$)$ & 0.038 \\
\hline $\begin{array}{l}\text { Resistive index } \\
\text { UIA } \\
\text { MIA } \\
\text { LIA }\end{array}$ & $\begin{array}{l}0.69(0.59 \text { to } 0.77) \\
0.69(0.605 \text { to } 0.79) \\
0.68(0.575 \text { to } 0.755)\end{array}$ & $\begin{array}{l}0.8(0.73 \text { to } 0.91) \\
0.85(0.735 \text { to } 0.935) \\
0.7(0.67 \text { to } 0.81)\end{array}$ & $\begin{array}{l}0.635(0.57 \text { to } 0.713) \\
0.625(0.593 \text { to } 0.715) \\
0.62(0.535 \text { to } 0.705)\end{array}$ & $\begin{array}{l}0.025 \\
0.02 \\
0.057\end{array}$ \\
\hline Kidney investigated area, $\mathrm{cm}^{2}$ & 49 (40.99 to 55.22$)$ & $54(52.24$ to 66.33$)$ & 45 (39.38 to 49.04$)$ & 0.02 \\
\hline WoAUC,a.u. & 7675 (4774 to 51225$)$ & 4884 (4629 to 6555$)$ & 24705 (6818 to 77384$)$ & 0.047 \\
\hline WiWoAUC,a.u. & $11544(7662$ to 76327$)$ & 7705 (6948 to 10022$)$ & $33475(10646$ to 111355$)$ & 0.031 \\
\hline $\begin{array}{l}\text { QoF, \% } \\
\text { Global graft } \\
\text { Cortical } \\
\text { Medulla }\end{array}$ & $\begin{array}{l}98(97 \text { to } 99) \\
96(91 \text { to } 98) \\
84(76 \text { to } 91)\end{array}$ & $\begin{array}{l}97(91 \text { to } 98) \\
93(83 \text { to } 95) \\
72(55 \text { to } 82)\end{array}$ & $\begin{array}{l}99(98 \text { to } 99) \\
97(93 \text { to } 98) \\
89(82 \text { to } 92)\end{array}$ & $\begin{array}{l}0.012 \\
0.025 \\
0.009\end{array}$ \\
\hline
\end{tabular}

The results are expressed as median (interquartile range, Q1 to Q3). UIA: Upper Interlobar Artery; MIA: Medium Interlobar Artery. LIA: Lower Interlobar Artery: WoAUC: wash-out area under the curve; WiWoAUC: wash-in and wash-out area under the curve; a.u.= acoustic units; QoF: Quality of fit; DGF: delayed graft function; EGF: early graft function

Table III. Association analysis in the early graft function (EGF) group: significant relationships

\begin{tabular}{ll}
\hline & $\begin{array}{l}\text { Spearman's } \\
\text { correlation } \\
\text { (p-value) }\end{array}$ \\
\hline Volume and global renal graft parameters & \\
WiAUC [a.u] & $0.58(0.047)$ \\
WiR [a.u] & $0.58(0.045)$ \\
WoAUC [a.u] & $0.6(0.038)$ \\
WiWoAUC [a.u] & $0.6(0.038)$ \\
Area [cm ${ }^{2}$ ] & $0.76(0.003)$ \\
Volume and cortical parameters & \\
PE [a.u] & $0.64(0.023)$ \\
WiAUC [a.u] & $0.7(0.01)$ \\
WiR [a.u] & $0.6(0.039)$ \\
WiPI [a.u] & $0.58(0.047)$ \\
WoAUC [a.u] & $0.64(0.024)$ \\
WiWoAUC [a.u] & $0.74(0.006)$ \\
WoR [a.u] & $0.64(0.025)$ \\
Volume and medulla parameters & \\
PE [a.u] & $0.69(0.012)$ \\
WiAUC [a.u] & $0.71(0.01)$ \\
WiR [a.u] & $0.63(0.028)$ \\
WiPI [a.u] & $0.69(0.012)$ \\
WoAUC [a.u] & $0.7(0.011)$ \\
WiWoAUC [a.u] & $0.7(0.011)$ \\
Volum and Area [cm ${ }^{2}$ ] association & $0.7(0.011)$ \\
\hline WiAUC: Wash-in area under the curve; WiR: &
\end{tabular}

WiAUC: Wash-in area under the curve; WiR: Wash-in Rate; WoAUC: Wash-out area under the curve; WiAUC+WoAUC: Wash-in and Wash-out AUC; PE:Peak Enhancement; WiAUC: Wash-in area under the curve; WiR:Wash-in Rate; WiPI: Wash-in Perfusion Index; WoR: Wash-out Rate; a.u: acoustic units.

necrosis (ATN). Vascular injuries that occur during rejection, affects small arterial vessels and cannot be properly assessed by Doppler ultrasonography alone [15] The superficial position of the renal graft and minimum organ movements allows better conditions to maintain a stable graft position for the assessment of the blood flow
Table IV. Association analysis for all patients: significant relationships.

\begin{tabular}{ll}
\hline & $\begin{array}{l}\text { Spearman's } \\
\text { correlation } \\
\text { (p-value) }\end{array}$ \\
\hline WoAUC [a.u] and GFR [ml/min] correlation & $0.51(0.03)$ \\
WiWoAUC [a.u] and GFR $[\mathrm{ml} / \mathrm{min}]$ & $0.53(0.024)$ \\
correlation & $0.58(0.012)$ \\
FT [s] and GFR [ml/min] correlation & $0.46(0.048)$ \\
QoF [\%] and area $\left[\mathrm{cm}^{2}\right]$ correlation & \\
\hline $\begin{array}{l}\text { WoAUC: Wash-out area under the curve; WiAUC: Wash-in area } \\
\text { under the curve; FT: Fall time; GFR: Gromerular filtrate rate; QoF: }\end{array}$ \\
quality of fit; a.u.= acoustic units
\end{tabular}

[16]. Resistive index (RI) provided by Doppler US, in the early post-transplant period is associated with acute tubular necrosis (ATN) and is a predictor for DGF and the development of chronic allograft nephropathy. However, the limitations of RI were proved by Cano et al who demonstrated that RI values are influenced by the status of the recipient vascularization [8]. In late US evaluation of the graft, increased RI is associated with acute rejection but not with ATN. No distinction can be made between humoral and cellular rejection based on RI alone [17]. Protocol biopsies are invasive and may be associated with severe complications and in DGF do not necessarily offer a benefit over risks [18]. In such cases, CEUS might provide an advantage and it may be used to avoid an unnecessary graft biopsy.

Evaluation of the renal graft by CEUS allows identification of surgical complications such as arterial and venous thrombosis, acute cortical necrosis, infectious complications, arterial stenosis and perirenal collections $[19,20]$. It also allows the identification of acute rejection and changes related to DGF $[21,22]$. 
In our study, DGF was associated with an increased recipient body mass index, increased graft volume, increased RI, decreased WoAUC and WiWoAUC. Lack of association between different CEUS parameters and RI suggests that CEUS might give a different insight compared with Doppler US over the graft vascularization. The only association to RI was found for cortical TTP in the DGF group. These findings seem to correspond to the general concept that TTP (as do the other time-related parameters such as RT, mTT and FT) describes the flow in the ROI, which should correlate with the resistance of the vessels, while intensity-related parameters (such as PE, WiAUC, WoAUC and WiWoAUC) describe the vasculature volume in the ROI [23].

The association between arterial FT and cortical WoAUC with GFR suggests that CEUS might be used for the evaluation of the glomerular function. Although, all the region of interest areas measured in the EGF group was positively associated with the QoF (meaning that the quality of TIC information depends on how much tissue is being "sampled"), such correlation was not found in the DGF group. We found that the larger the ROI area, the better the QoF. However, even if the graft with delayed function was larger and as a consequence the ROI area, the QoF was lower in the DGF group compared with the EGF group. Such a difference is difficult to explain but it may suggest the existence of more complex hemodynamical processes in the DGF group than in the EGF group. Moreover, such differences are more pronounced in the medulla.

ROI area, directly linked to the size of the kidney, might be a source of bias and further studies are needed for standardization. Other limits of our study include a relatively small sample size, lack of information concerning the systemic blood flow of patients (with a possible influence on CEUS behavior) and the limited availability of morphological data needed for a proper differentiation of the causes of DGF. Since DGF has many similitudes with ATN, CEUS evaluation of such grafts might be useful to differentiate between the two situations. After excluding irreversible causes of DGF (cortical necrosis and renal artery or venous thrombosis)CEUS may be useful for the monitoring of the graft outcome.

\section{Conclusions}

Intensity CEUS parameters WoAUC and WiWoAUC may be useful to diagnose and follow-up grafts with delayed function. Lack of association between different CEUS parameters and RI suggests that CEUS might give a different insight compared with Doppler US over the graft vascularization. CEUS investigation might be used for the estimation of the glomerular function due to the association between arterial FT and cortical WoAUC with GFR. Additional studies on larger cohorts are required for confirmation of the CEUS utility in the routine evaluation of the transplanted kidney.

\section{Conflict of interest: none}

Acknowledgment. This work was supported by a grant from the Ministry of Research and Innovation, CNCSIS - UEFISCDI, project number PN-III-P4-IDPCE-2016-0701, with PNCDI III, contract number: $184 / 2017$.

\section{References}

1. Fischer T, Dieckhofer J, Muhler M, et al. The use of contrast-enhanced US in renal transplant: first result and potential clinical benefit. Eur Radiol 2005;15 Suppl 5:E109E116.

2. Kadambi PV, Brennan DC, Chon J. Kidney transplantation in adults: Evaluation and diagnosis of the patient with renal allograft dysfunction. .UpToDate. Available from: https:// www.uptodate.com/contents/kidney-transplantation-inadults-evaluation-and-diagnosis-of-the-patient-with-renalallograft-dysfunction\#H3674584560

3. Wang CJ, Tuffaha A, Phadnis MA, Mahnken JD, Wetmore JB. Association of slow graft function with long-term outcomes in kidney transplant recipients. Ann Transplant 2018;23:224-231.

4. Humar A, Johnson EM, Payne WD, et al. Effect of initial slow graft function on renal allograft rejection and survival. Clin Transplant 1997;11:623-627.

5. Schröppel B, Legendre C. Delayed kidney graft function: from mechanism to translation. Kidney Int 2014;86:251258.

6. Bahl D, Haddad Z, Datoo A, Qazi YA. Delayed graft function in kidney transplantation. Curr Opin Organ Transplant 2019;24:82-86.

7. Kidney Disease: Improving Global Outcomes (KDIGO) Transplant Work Group. KDIGO clinical practice guideline for the care of kidney transplant recipients. Am J Transplant 2009;9 (Suppl 3):S1-S157.

8. Cano H, Castañeda DA, Patiño N, et al. Resistance index measured by doppler ultrasound as predictor of graft function after kidney transplantation. Transplant Proc 2014;46:2972-2974.

9. Oon SF, Foley RW, Quinn D, Quinlan DM, Gibney RG. Contrast-enhanced ultrasound of the kidney: a single-institution experience. Ir J Med Sci 2018;187:795-802.

10. Cai R, Tao L, Liang W, Lin M, Guo X, Huang XE. Application of Contrast-Enhanced Sonography on the Diagnosis of Acute and Chronic Rejection After Renal Transplantation. Ultrasound Q 2020;36:59-63.

11. Cokkinos DD, Antypa EG, Skilakaki M, Kriketou D, Tavernaraki E, Piperopoulos PN. Contrast Enhanced Ultra- 
sound of the Kidneys: What Is It Capable of? Biomed Res Int 2013;2013:595873.

12. Dietrich CF, Averkiou M, Nielsen MB, et al. How to perform Contrast-Enhanced Ultrasound (CEUS). Ultrasound Int Open 2018;4:E2-E15.

13. D’Onofrio M, Crosara S, De Robertis R, Canestrini S, Mucelli P. Contrast-Enhanced Ultrasound of Focal Liver Lesions. AJR Am J Roentgenol 2015;205:W56W66.

14. Benozzi L, Cappelli G, Granito M, et al. Contrast-enhanced sonography in early kidney graft dysfunction. Transplant Proc 2009;41:1214-1215.

15. McArthur C, Baxter GM. Current and potential renal applications of contrast-enhanced ultrasound. Clin Radiol 2012;67:909-922.

16. Zeisbrich M, Kihm LP, Druschler F, Zeier M, Schwenger $\mathrm{V}$. When is contrast-enhanced sonography preferable over conventional ultrasound combined with Doppler imaging in renal transplantation? Clin Kidney J 2015;8:606614.

17. Preuss S, Rother C, Renders L, et al. Sonography of the renal allograft: Correlation between doppler sonographic resistance index (RI) and histopathology. Clin Hemorheol Microcirc 2018;70:413-422.
18. Favi E, James A, Puliatti C, et al. Utility and safety of early allograft biopsy in adult deceased donor kidney transplant recipients. Clin Exp Nephrol 2019 Nov 25. doi: 10.1007/ s10157-019-01821-7.

19. Álvarez Rodríguez S, Hevia Palacios V, Sanz Mayayo E, et al. The Usefulness of Contrast-Enhanced Ultrasound in the Assessment of Early Kidney Transplant Function and Complications. Diagnostics (Basel) 2017;7:E53.

20. Putz FJ, Erlmeier A, Wiesinger I, et al. Contrast-enhanced ultrasound (CEUS) in renal imaging at an interdisciplinary ultrasound centre: Possibilities of dynamic microvascularisation and perfusion. Clin Hemorheol Microcirc 2017;66:293-302.

21. Grzelak P, Szymczyk K, Strzelczyk J, et al. Perfusion of kidney graft pyramids and cortex in contrast-enhanced ultrasonography in the determination of the cause of delayed graft function. Ann Transplant 2011;16:48-53.

22. Jin Y, Yang C, Wu S, et al. A novel simple noninvasive index topredict renal transplant acute rejection by contrast-enhanced ultrasonography. Transplantation 2015;99:636-641.

23. Dietrich CF, Averkiou MA, Correas JM, Lassau N, Leen E, Piscaglia F. An EFSUMB introduction into Dynamic Contrast-Enhanced Ultrasound (DCE-US) for quantification of tumour perfusion. Ultraschall Med 2012;33:344-351. 